\title{
Reliblen TCP based Communication in Mobile Ad hoc Network (MANET)
}

\author{
Ankit Kumar Agrawal ${ }^{1}$, Prof. Damodar Tiwari ${ }^{2}$ \\ Bansal Institute of Science \& Technology, Bhopal, India ${ }^{1}$ \\ Assistant Professor, Bansal Institute of Science \& Technology, Bhopal, India ${ }^{2}$
}

\begin{abstract}
The function of transport layer is to maintain the end to end synchronization in between sender to receiver. In this layer of network basically only one reliable protocol is working because of its having the concept of ACK (Acknowledgement). In Mobile Ad hoc Network the structure of network is not possible to define because the mobile nodes are continuously moves in limited area. In this paper we proposed the new approach of routing based on data rate and queue control scheme applied on TCP Newreno, TCP Reno and TCP Westwood protocol in network. The proposed work maintains the reliability under MANET and minimized the network delay, overhead, congestion, collision and other drop reasons. In this research we compare the performance of proposed work with existing the proxy acknowledge mechanism provide efficient TCP communication in the MANET environment and normal AODV routing with TCP variants. In Proxy technique proxy node store the information about data drop reasons and resolve some dropped condition. Through proposed approach we increased the network reliability with the help of TCP variant technique utilized under mobile Ad hoc network. The proposed scheme performance is better than normal and proxy method.
\end{abstract}

Keywords: MANET, TCP Variants, AODV, Proxy, Proposed scheme, Queue, Congestion and collision.

\section{INTRODUCTION}

Wireless communications offer versatile, transportable and low value communication and accumulated productivity and potency. Wireless native space network (WLAN) devices, as an example, enable users to manoeuvre their laptops from place to put among their offices while not the necessity for wires and while not losing network property. Less wiring suggests that larger flexibility, accumulated potency, and reduced wiring prices. Unintentional networks, like those enabled by Bluetooth while not want of central or static devices, enable information synchronization with network systems and application sharing between devices. Hand hold devices like personal digital assistants (PDA) and cell phones enable remote users to synchronize personal databases and supply access to network services like wireless e-mail, internet browsing, and net access. Moving bulk information quickly over high-speed information network may be a demand for several applications. These applications need high-bandwidth links between network nodes [1].The application of the mobile unintentional network includes military field circumstance, disaster relief, and rescue throughout emergency, discovery etc. The cluster connected application of mobile unintentional networks embody conference, information dissemination services etc [2].

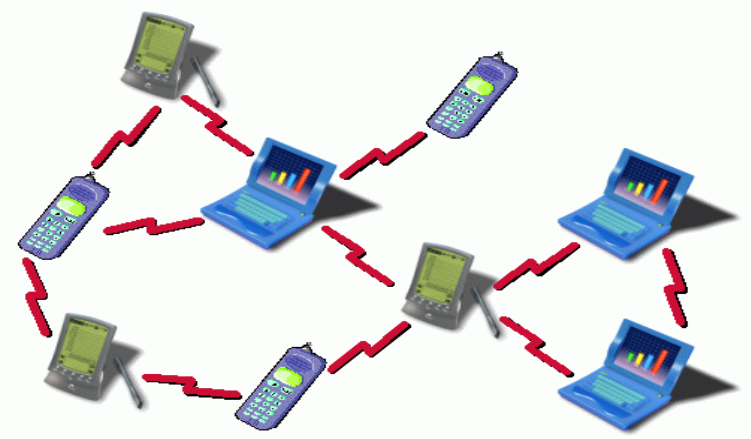

Figure 1.1: Mobile ad-hoc Network

MANETs is an IEEE 802.11 framework. It is an interconnected collection of wireless nodes where there is no networking infrastructure in the form of base stations, devices do not need to be within each other's communication range to communicate, the end-users devices also act as routers, nodes can enter and leave over time, data packets are forwarded by intermediate nodes to their final destination. 
A Mobile Ad-hoc Network (MANET) may be a network consisting of a set of nodes capable of human action with one another while not facilitate from a network infrastructure. Applications of MANETs embody the field applications, rescue work, additionally as civilian applications like an out of doors meeting, or an ad-hoc room. With the increasing range of applications to harness the benefits of unexpected Networks, additional considerations arise for security problems in MANETs. The routing protocols [3][4] in MANET is also different from other network protocols. In this paper the main focus on to improve the performance of different TCP variants protocols mainly discuss in this paper like TCP Newreno, TCP Reno and TCP Westwood [5][6]. The routing technique used is AODV in MANET.

\section{LITERATURE SURVEY}

Several researchers have done the quantitative and qualitative analysis of ad hoc Routing Protocols by suggests that of various performance parameters. Conjointly they need used completely different simulators for this purpose.

May Zin Oo, Mazliza Othman, and Timothy O'Farrell [1] "A Proxy Acknowledgement Mechanism for protocol Variants in Mobile unplanned Networks" during this title a sequence variety checking technique is projected to enhance the performance of protocol connections in mobile unplanned networks. Whereas a protocol association is initialized, a routing protocol takes the responsibility for checking the hop count between a supply and destination combine. If the hop count is bigger than a predefined worth, the routing protocol decides to use a proxy node. The Responsibility of a proxy node is to envision the correctness of knowledge packets associate degreed inform the missing packets by causation an acknowledgement from a proxy node to the supply node. By doing therefore, the supply node is in a position to channel associate degree missing packet prior to while not waiting till an end-to-end acknowledgement is received from the destination.

Breeson Francis et al [7] presents varied performance improvement mechanisms by that protocol doesn't think about each packet loss as congestion. These mechanisms facilitate protocol to tell apart between congestion and packet loss and increase turnout performance.

Purvang Dalal et. Al. [8] during this title a combine of approaches to form protocol adaptable to wireless state of affairs and improve the end-to-end performance. Grades of intensive simulations demonstrate important performance improvement of protocol with the projected modifications over the traditional protocol, once the network is littered with frequent link failures

M.I Tayade et al. [9] gift some approaches to enhance the protocol performance over mobile unplanned networks. The principal downside of protocol in MANET's surroundings is clearly its incapacity to tell apart between losses iatrogenic by network congestion et al sorts of losses. Protocol assumes that losses square measure perpetually thanks to network congestion. a lot of analysis is to be worn out future to explore the result of all this ways on different protocol variants.

Adel Gaafar A. Elrahim et al. [10] describes a modification to the transmission \{control protocol|TCP |protocol communications protocol $\}$ congestion control to be used in wireless device networks. It shows that by slightly mediating the algorithmic rule of the protocol, it is created to retort higher to wireless links, whereas maintaining its benefits on the wired networks at an equivalent time. this can be definitely a solely fascinating feature because the standard protocol in most cases conducts the stress of the "Wireless links of the network. That the changed protocol provides higher performance in terms of upper turnout, delay, retransmission, and energy consumption than standard protocol protocol.

Meng Xiao-jing et al. [11] projected associate degree improved algorithmic rule TCPW-M, the new algorithmic rule through RTT within the sample area interference changes to completely different congestion levels and improve the information measure estimation algorithmic rule in step with the various congestion levels. Finally, the simulation analysis proves that the improved algorithmic rule will increase the soundness of the information measure estimates.

Vishnu Kumar Sharma and Dr. Sarita Singh Bhadauria [12] "Performance Analysis on Mobile Agent based mostly Congestion management mistreatment AODV Routing Protocol Technique with Hop by Hop algorithmic rule for Mobile Ad-hoc Network" during this paper, we tend to propose to agent based mostly congestion management technique for MANETs. In our technique, the data regarding network congestion is collected and distributed by mobile agents (MA) A mobile agent based mostly congestion management AODV routing protocol is projected to avoid congestion in unplanned network. Some mobile agents square measure other in unplanned network, that carry routing data and nodes congestion standing. once mobile agent travels through the network, it will choose a less-loaded neighbor node as its next hop and update the routing table in step with the node's congestion standing.

P.Vigneshwaran, Dr. R. Dhanasekaran [13] "A Novel Protocol to enhance protocol Performance -Proposal" during this title a survey is dole out to spot the analysis problems associated with all layers which can facilitate in developing a routing protocol to enhance the protocol performance.

Sofiane Hamrioui , Mustapha Lalam, Pascal Lorenz [14] "IA-TCP: rising Acknowledgement Mechanism of protocol for higher performance in MANET" during this paper, we recommend associate degree improvement of the Transmission management Protocol (TCP), referred to as Improvement of the Acknowledgement mechanism of protocol (IA-TCP), to higher performance in painter. IA-TCP delays the acknowledgements packets of protocol in step with the amount of nodes, their ability and also the communication distance between these nodes. we tend to restricted our studies on vital 
parameters in such networks that square measure turnout and end-to-end delay as a result of they need nice effects on the protocol performance in painter. The results square measure satisfactory and showed that IA-TCP will outmatch not solely protocol customary, however conjointly similar techniques that are projected.

\section{PROBLEM STATEMENT}

The Wireless mobile Ad-hoc network is a recent communication environment whose provide any where any time communication in dynamic routing manner, because huge number of mobile nodes spread over short distances. MANET routing strategies is challenging task because node frequently change their position and routing table change while node update the position. From the previous research papers we indentify the problem of network reliability as well as data reliability. Some researcher modified the routing strategies and takes the decision to provide more reliable communication and some of researcher work in the field of data reliability and form that they use TCP based communication. In our thesis dissertation aim to design reliable data service using data dropping reason identification and path reliability based communication. That problem statement provide the direction to our researcher and after the achieving the goal we compare our work through exiting methodology.

\section{PROPOSED SECURITY SCHEME AGAINST SYBIL ATTACK}

In this section describe the proposed algorithm, where input parameters set as number of nodes, intermediate nodes, destination and sender nodes etc. after the all input declaration AODV routing protocol are execute and select the route from source to destination and then send the TCP data, during that time sender sends the data through intermediate nodes. while the data drop by collision than CTS/RTS message resolve the problem of collision using priority assignment of each senders that priority message generate by the proxy node and sends to all sender so that collision not arises in the network, similarly other data drop reasons are resolve by the proxy node so in future data drop are minimized and quality of service of the network improve.

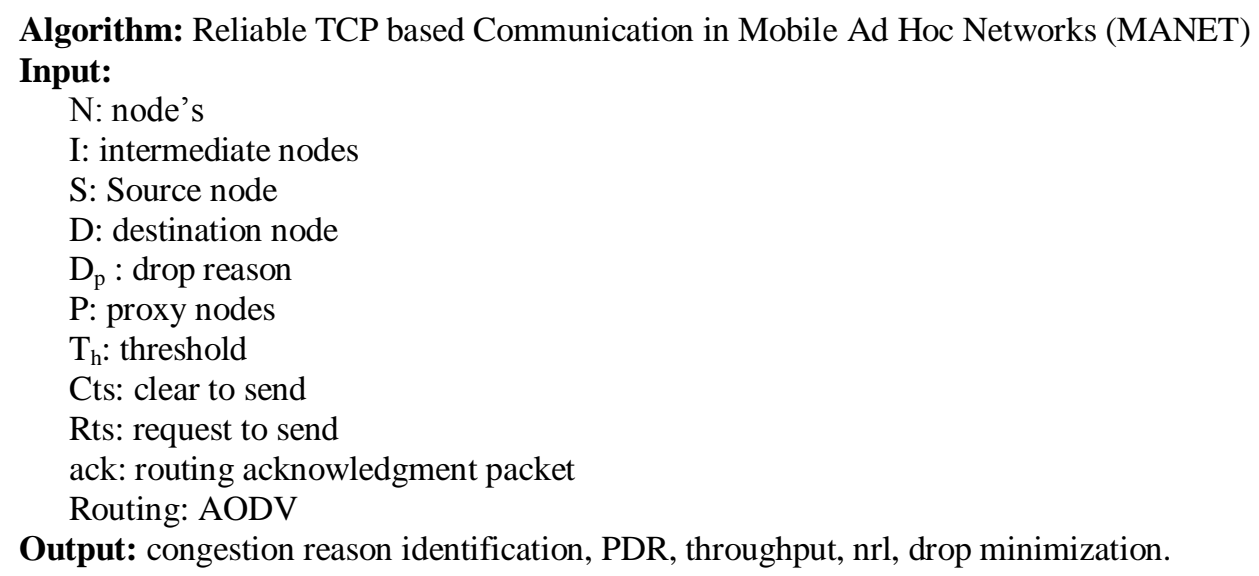

\section{Procedure:}

Step1:S call (AODV)

$\operatorname{AODV}\left(\mathrm{S}, \mathrm{D}, \mathrm{r} \_\mathrm{pkt}\right)$

Step2: I receives AODV packet

I check $D$ is

If $\mathrm{D}$ id != I id then

$\mathrm{I} \leftarrow$ prepare fresh routing table

Forward (AODV) to next hop

Else

Goto step 2

$D$ found

$\mathrm{D} \leftarrow$ sends ack to $\mathrm{S}$ node by reverse path

End if

Call (data_pkt)

Step3: $S$ generate data packets

data_pkt(S, D, data_type)

I receive data_pkt 
Step 4: If I receive data_pkt $>$ one $S \& \&$ time $==$ same then

$\mathrm{D}_{\mathrm{p}}$ by collision

Call (AODV, Cts/Rts)

Assign priority of $\mathrm{S}$ nodes

$\mathrm{P}$ send priority message to all $\mathrm{S}$ node

Else if I receives data_pkt $\& \&$ data drop then

$\mathrm{P} \leftarrow$ identifies drop reason

If reason $==$ queue full $\mid$ congestion then

$\mathrm{P} \leftarrow$ check the $\mathrm{Q}$ limit

If $\mathrm{Q}$ limit $>\mathrm{T}_{\mathrm{h}}$ then

End if $\mathrm{Q} \leftarrow \mathrm{Q}+1$

$\mathrm{P}$ calculate delay

If delay $>$ max then

Search fresh route

\section{End if}

Else if reason $==$ node motion then

$\mathbf{P}$ execute local route repair

$\mathbf{P}$ sends fresh route info to $\mathrm{S}$ node

End if

End if

Step 5: If I receive data_pkt \&\& forward to D node then

Calculate delay, PDR and throughput

End if

\section{SIMULATION TOOL OVERVIEW}

The network simulator (ns-2) is a discrete network simulator targeted at network researching. Ns-2 originated in 1989 as a variant of the REAL network simulator. As a part of the Virtual Internetworks Test bed (VINT) project at the University of California in Berkley. The project was supported by Defense Advanced Research Projects Agency (DARPA) in 1995 [15]. Ns-2 is an object-oriented simulator with substantial support available for simulation of TCP, routing, and multicast protocols, initially intended for wired networks, but the Monarch Group at Carnegie Mellon University (CMU) have extended ns-2 to support wireless networks. The core of the simulator, including the network protocols is implemented in $\mathrm{C}++$, while object oriented TCL is used as an interface to describe, and set up the simulations. The implementation of ns-2 closely follows the OSI model. The essential of the wireless model consist the Mobile Node at the core, with additional support for simulations of multi-hop ad-hoc network etc.

\section{A. Simulation Parameter:}

Simulation parameters is used is simulation is mentioned in table 1.

According to these simulation parameters the performance of three protocols i.e. Newreno, Reno and Westwood are evaluated with different routing mechanism. The performance of normal AODV routing, Proxy and proposed with these three transport layer protocol is evaluated and observe that the performance of proposed routing with TCP Newreno, TCP Reno and proposed is provides better performance.

Table.1 Simulation Parameters

\begin{tabular}{|l|l|}
\hline Simulator Used & NS-2.31 \\
\hline Number of nodes & 30 \\
\hline Dimension of simulated area & $800 \mathrm{~m} \times 800 \mathrm{~m}$ \\
\hline Routing Protocol & AODV \\
\hline Methodology & Proxy \\
\hline Simulation time & 100 sec. \\
\hline TCP Type & Newreno, Reno, Westwood \\
\hline Packet size & 1024 bytes \\
\hline Number of traffic connections & 12 \\
\hline Node movement at maximum Speed & Random \\
\hline Transmission range & $550 \mathrm{~m}$ \\
\hline
\end{tabular}




\section{RESULT ANALYSIS}

In this section result analysis of different TCP protocol with proposed approach is simulate. The performance of proposed scheme is provides better results.

\section{A. Packet Delivery Ratio Analysis (TCP-Newreno)}

In this graph the performance of routing with transport layer protocol TCP Newreno is evaluated and recognizes that the proposed congestion and collision control scheme provides better results. The proposed protocol performance is about provides 99\% successful data delivery in network. Due to minimizes congestion in network routing performance is provides better results. The routing performance of proposed scheme is provides the better results and also handle network load.

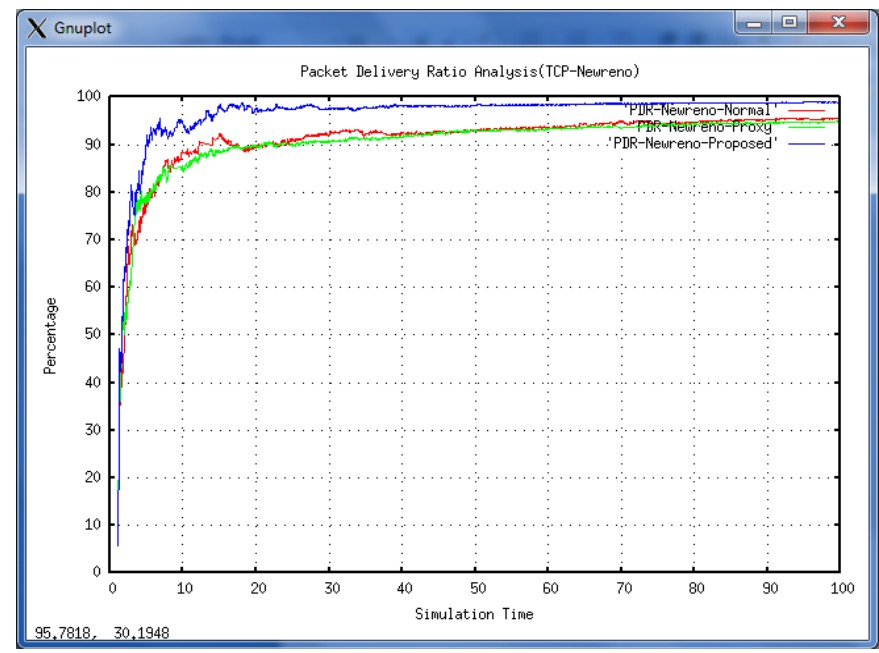

Figure 2 PDR Analysis of TCP Newreno

B. Packet Delivery Ratio Analysis (TCP-Reno)

In this graph the performance of routing with transport layer protocol TCP Reno, is evaluated and recognize that the proposed scheme is provides better results. The proposed protocol performance is about provides $99 \%$ successful data delivery in network. Due to minimizes congestion in network routing performance is provides better results. The packet percentage ratio shows the possibility successful data deliver at destination. The congestion and collision is occurring due to heavy load and load is controlled by proposed routing performance.

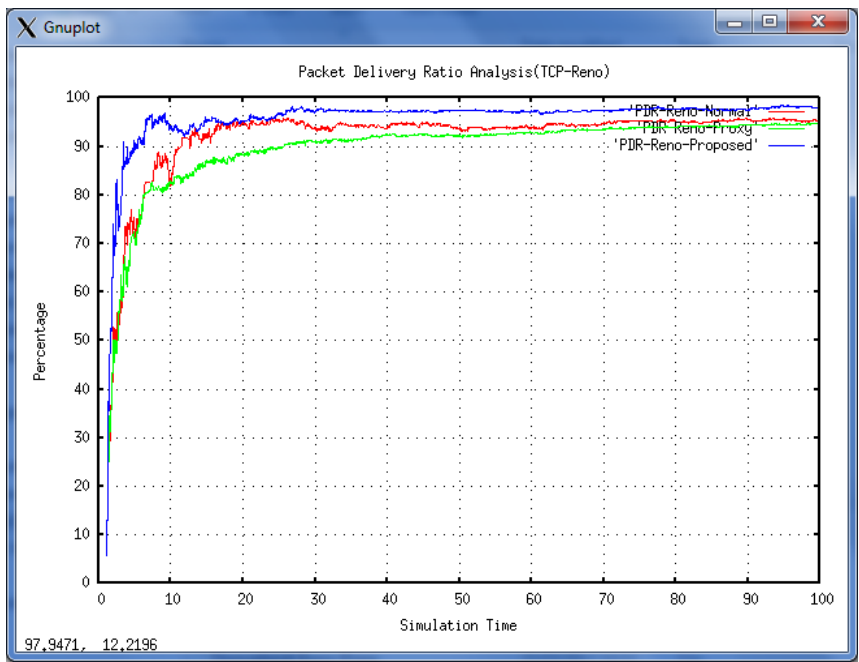

Figure 3 PDR Analysis of TCP Reno

C. Packet Delivery Ratio Analysis (TCP-Westwood)

The TCP Westwood is another TCP protocol to maintain better end to end communication in dynamic network in this graph the performance of TCP Westwood protocol is evaluated and recognize that the proposed scheme is provides 
better results. The proposed protocol performance is about provides $99 \%$ successful data delivery in network. Due to minimizes congestion in network routing performance is provides better results. The packet percentage ratio shows the possibility of successful data deliver at destination. The congestion and collision is occurring due to heavy load and load is controlled by proposed routing scheme efficiently.

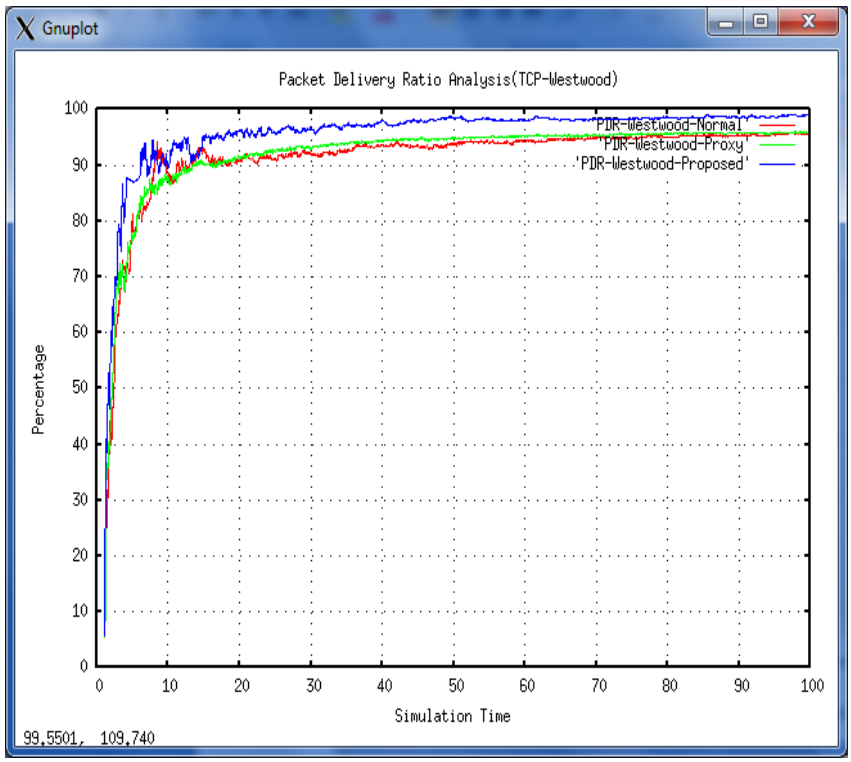

Figure 4 PDR Analysis of TCP Westwood

\section{Throughput Analysis (TCP-Newreno)}

In throughput, performance is evaluated and recognizes the performance of TCP Newreno in per unit of time in network. The proposed congestion control performance is provides highest data receiving in per unit of time in network. Due to minimizes congestion in network routing performance is provides better results. The proposed throughput performance is about more than 1500 at time 28 seconds and maintained at $1100 \mathrm{pks} / \mathrm{sec}$ up to end of simulation time. That shows the proposed scheme is efficiently handled heavy load in network.

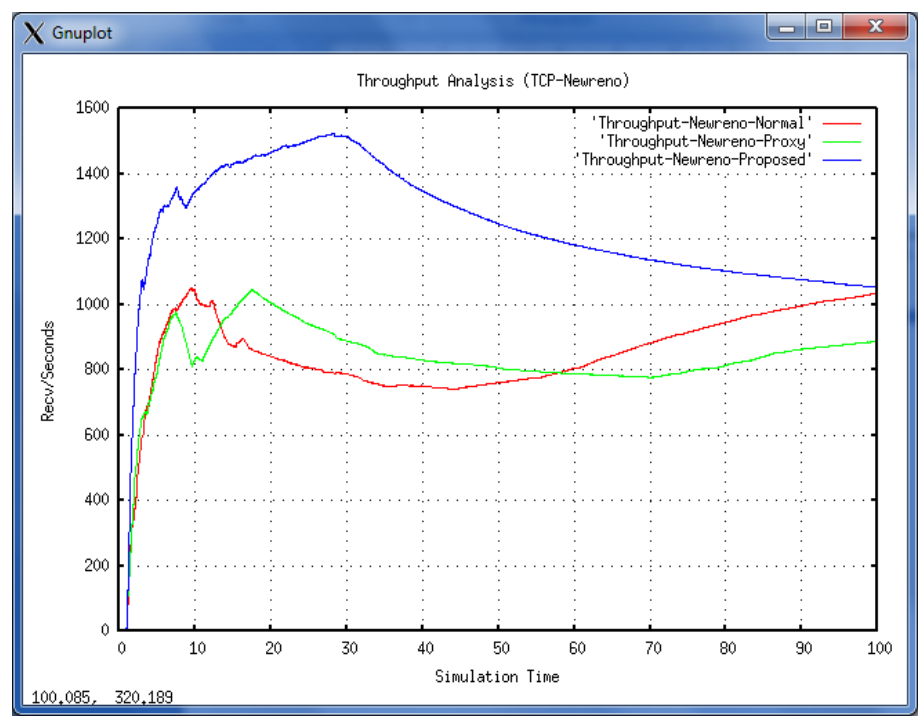

Figure 5 Throughput Analysis of TCP Newreno

\section{E. Throughput Analysis (TCP-Reno)}

In throughput, performance is evaluated and recognizes the performance of TCP Reno in per unit of time in network. The proposed congestion control routing performance is provides highest data receiving in per unit of time in network. the performance of routing in TCP Reno is much better than Westwood. The proposed throughput performance is about 1500 at time 28 seconds and maintained at $1200 \mathrm{pks} / \mathrm{sec}$ up to end of simulation time. Only vary in time between 40 to 60 seconds. That shows the proposed scheme is efficiently handled heavy load in network. 


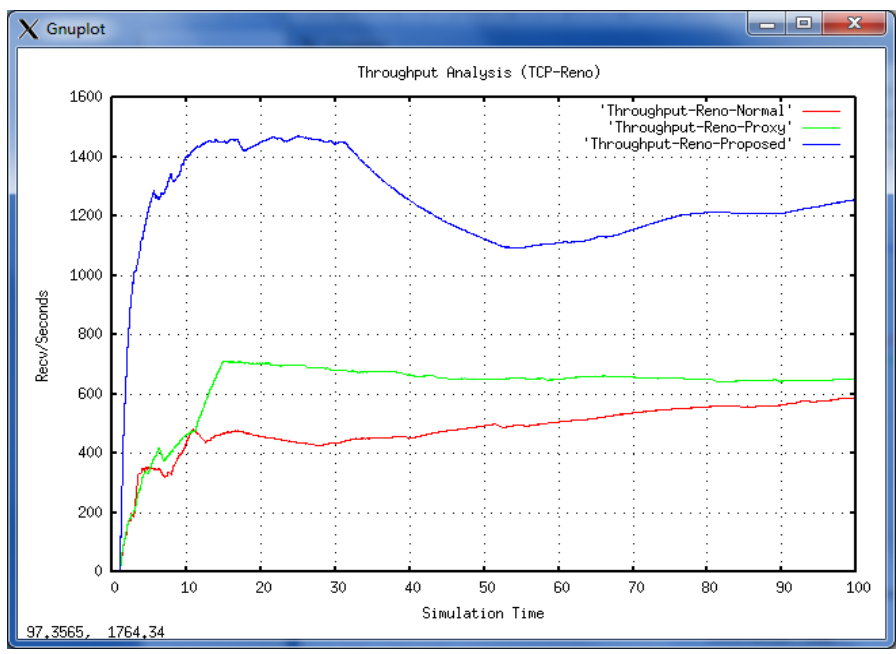

Figure 5 Throughput Analysis of TCP Reno

F. Throughput Analysis (TCP-Westwood)

The throughput performance TCP Wetwood is measured in per unit of time in network. The proposed congestion protocol performance is provides highest data receiving in per unit of time in network. Due to minimizes congestion in network routing performance is provides better results. The proposed throughput performance is about more than 1500 but at the start of simulation performance is degrades and counted about 800 for 10 seconds but after that continuously enhanced in network.. The proposed scheme is efficiently knob heavy load in network.

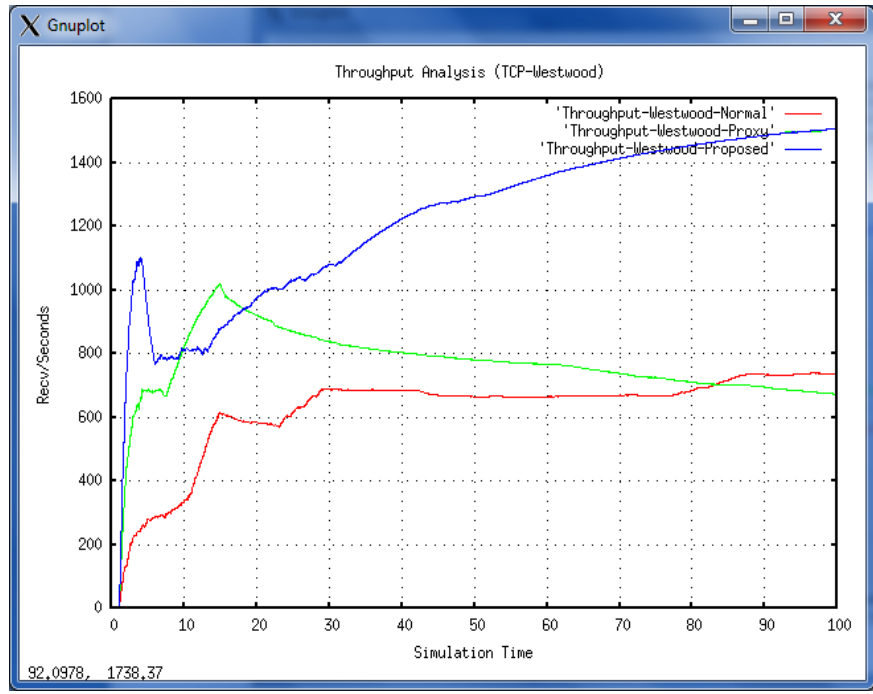

Figure.7 Throughput Analysis of TCP Westwood

\section{CONCLUSION AND FUTURE WORK}

TCP (Transmission Control Protocol) is the most reliable protocol for communication but it is also time consume in confirmation of data delivery through ACK. TCP performs poorly in such networks where the possibility of congestion and collision is occur and because of that packet loss is measured in good quantity. In this paper, we point out the major reasons for the performance degradation and provides proposed scheme for resolution. The factors such as mobility affect the TCP performance over single and multi hop ad hoc networks, while, aside from these factors, other factors such as medium access contention, frequent route changes, and breakages are considered to lead to the poor TCP performance. In this proposed work the packet dropping is minimizes and performance of network is improved. The TCP variants consider in simulation like TCP Newreno, TCP Reno and TCP Westwood with normal AODV routing, Routing with existing Proxy mechanism and proposed data rate control with queue handling in MANET. The proposed scheme with different TCP variants provides better results. The simulation results evaluated through different performance metrics and in these metrics provide better results in case of proposed scheme. The throughput and PDR metrics are gives better results with each TCP variant with proposed congestion and collision control scheme in MANET. 
The TCP is reliable protocol but in heavy loaded network every protocol is in trouble and not provides complete performance in network. In future we proposed the algorithm based on load. The data is deliver through path where data rate is minimum or in previous data forwarding is poor but success rate is considerable.

\section{REFERENCES}

[1] May Zin Oo, Mazliza Othman, and Timothy O’Farrell “A Proxy Acknowledgement Mechanism for TCP Variants in Mobile Ad Hoc Networks" Journal Of Communications And Networks, Vol. 18, NO. 2, April 2016.

[2] F. Alam, "Node feed-back based TCP scheme for Mobile Ad hoc Networks", GESJ: Computer Science and Telecommunications, No 2 (31), 2011.

[3] Elizabeth Belding-Royer, "Routing approaches in Mobile Ad hoc Networks", in: S. Basagni, M. Conti, S. Giordano, I. Stojmenovic (Eds.), Ad Hoc Networking, IEEE Press Wiley, New York, 2003.

[4] C. Perkins, E. Belding-Royer, S. Das, "Ad hoc On-Demand Distance Vector (AODV) Routing”, Network Working Group Request for Comments: 3561, 2003

[5] Harpreet Singh Chawla, M. I. H. Ansari, Ashish Kumar, Prashant Singh Yadav “A Survey of TCP over Mobile AD-HOC Networks" International Journal of Scientific \& Technology Research Volume 1, Issue 4, May 2012.

[6] Md Nazmul Islam Khan, Rashed Ahmed and Md. Tariq Aziz, " A Survey of TCP Reno, New Reno and Sack Over Mobile Ad-Hoc Network",International Journal of Distributed and Parallel Systems (IJDPS) Vol.3, No.1, January 2012.

[7] Breeson Francis, Venkat Narasimhan, Amiya Nayak, Ivan Stojmenovic "Techniques for Enhancing TCP Performance in Wireless Networks" 2012 32nd International Conference on Distributed Computing Systems Workshops Canada.

[8] Purvang Dalal, Nikhil Kothari and K. S. Dasgupta "Improving TCP Performance over Wireless Network with Frequent Disconnections" International Journal of Computer Networks \& Communications (IJCNC) Vol.3, No.6, November 2011.

[9] Mandakini Tayade, Sanjeev Sharma, "Performance improvement of tcp in manets" International Journal of Engineering Science and Technology (IJEST).

[10] Adel Gaafar A.Elrahim, Hussein A.Elsayed, Salwa Ramlf, Magdy M, Ibrahim "Improving TCP Congestion Control for Wireless Sensor Networks"IEEEXplore page1-6 14-17 June 2011.

[11] MENG Xiao-jing, LIU Jin, WANG Li-li "Research on TCPW improvement in hybrid network" Computer Science and Automation Engineering (CSAE), 2011 IEEE International Conference on 10-12, pp. 650-653 June 2011.

[12] Vishnu Kumar Sharma and Dr. Sarita Singh Bhadauria [9] "Performance Analysis on Mobile Agent Based Congestion Control Using AODV Routing Protocol Technique with Hop by Hop Algorithm for Mobile Ad-hoc Network" International Journal of Ad hoc, Sensor \& Ubiquitous Computing (IJASUC) Vol.3, No.2, April 2012.

[13] P.Vigneshwaran, Dr. R. Dhanasekaran [10] "A Novel Protocol to Improve Tcp Performance -Proposal" International Journal of Computer Engineering \& Technology (IJCET) Volume 3, Issue 2, July- September (2012), pp. 372-377.

[14] Sofiane Hamrioui, Mustapha Lalam, Pascal Lorenz "IA-TCP: Improving Acknowledgement Mechanism of TCP for better performance in MANET" The Society of Digital Information and Wireless Communications, 2012.

[15] http://www.isi.edu/nsnam/ns/ 